\title{
Occupational therapists' perceptions of service transformation towards contemporary philosophy and practice in an acute specialist paediatric hospital
}

Short title: Contemporary philosophy and practice in a paediatric hospital

1. Alice Murray, former Student Occupational Therapist, Discipline of Occupational Therapy, School of Allied Health Sciences, Griffith University, Queensland, Australia; Occupational Therapist, Occupational Therapy and Music Therapy, Queensland Children's Hospital, Queensland Hospital and Health Service, Queensland, Australia

2. Amelia Di Tommaso, Lecturer, Discipline of Occupational Therapy, School of Allied Health Sciences, Griffith University, Queensland, Australia

3. Matthew Molineux, Professor and Head, Discipline of Occupational Therapy, School of Allied Health Sciences, Griffith University, Queensland, Australia

4. Anna Young, Director, Occupational Therapy and Music Therapy Department, Queensland Children's Hospital, Queensland Hospital and Health Service, Queensland, Australia

5. Penny Power, Acting Director, Occupational Therapy and Music Therapy Department, Queensland Children's Hospital, Queensland Hospital and Health Service, Queensland, Australia; Clinical Education Leader - Paediatrics, Occupational Therapy Clinical Education Program, Queensland Health, Australia

\section{Corresponding Author:}

Dr Amelia Di Tommaso

Discipline of Occupational Therapy, School of Allied Health Sciences, Griffith University, Parklands Drive, Southport, 4222, Queensland, Australia

Email: a.ditommaso@griffith.edu.au 


\begin{abstract}
Introduction: The acute occupational therapy department at a specialist paediatric hospital in metropolitan Australia, is undergoing a service transformation to increase their alignment with contemporary occupational therapy philosophy and practice. The purpose of this study was to explore occupational therapists' current knowledge and skills regarding contemporary occupational therapy philosophy and practice, and their attitudes and motivation towards a service transformation.
\end{abstract}

Method: Qualitative data was collected through semi-structured interviews with eight occupational therapists. Interviews were audio-recorded and transcribed for thematic data analysis.

Findings: Four major themes were established: a glimmer of occupation, variability with recognising and articulating the core of occupational therapy, externalising challenges, and the anticipated costs and benefits of the transformation.

Conclusion: The findings suggest that therapists in acute settings are attempting to incorporate contemporary occupational therapy philosophies in practice. Therapists recognised the power of occupation and the benefits of an occupation-based approach, hence were accepting of the service transformation, however the implementation of occupationbased practice appears to be inconsistent in acute settings. Findings highlight a need to enhance therapists' knowledge of contemporary occupational therapy. However, therapists did not foresee this as a significant factor, leading to feeling a lack of control and externalising the barriers to service transformation.

KEY WORDS occupational therapists, occupational therapy philosophy, occupation-based practice, acute hospital setting 


\section{Introduction and literature review}

Over the past two decades the call for occupational therapy practice to better reflect the contemporary philosophies of the profession has increased (Gustafsson et al., 2014; Kielhofner, 2009; Wilding and Whiteford, 2007; Yerxa, 1992). Paradigm shifts throughout the professions' history have meant that occupational therapists have been largely influenced by a biomedical model focusing on impairment, resulting in a move away from the core philosophies which the profession was founded upon (Kielhofner, 2009). As a result, there was a call for a paradigm shift in the 1960s, with the purpose of re-establishing a focus on occupation as the core domain (Yerxa, 1992; Kielhofner, 2009). Therefore, the Contemporary Paradigm emerged with three central constructs; "the importance of occupation to health and well-being, recognition of occupational problems/challenges and occupation-based practice" as the approach to therapy (Kielhofner, 2009: 45). While Kielhofner (2009) claimed that the profession moved towards the Contemporary Paradigm from the 1960's, other authors question whether a contemporary approach has been adopted by all members of the profession (Gillen and Greber, 2014; Gustafsson et al., 2014; Parnell and Wilding, 2010).

One construct which occupational therapists particularly struggle with implementing is occupation-based practice (Di Tommaso et al., 2016; Keesing and Rosenwax, 2011; Wilding and Whiteford, 2007), which is a defining element of Kielhofner's (2009) Contemporary Paradigm. Furthermore, there is a lack of agreement about what constitutes occupation-based practice which may explain the reason for these difficulties (Fisher, 2013). For this review, occupation-based practice was defined as "engaging clients in occupations as the therapeutic modality of change, specifically in the evaluation and intervention stages" (Fisher, 2013: 98). Moreover, occupational therapists in acute hospital settings have particular difficulty implementing occupation-based practice (Di Tommaso et al., 2016; Wilding and Whiteford, 2007) due to a range of contextual factors such as time pressures, the 
biomedical model and discharge focus, as well as individual factors such as identity confusion and difficulty articulating the occupational therapy role (Britton et al., 2016).

Some studies of occupational therapists in acute settings have considered how their practice can be more congruent with contemporary occupational therapy perspectives. For example, Wilding and Whiteford $(2008 ; 2009)$ explored the use of occupation in acute settings through their action research study. Their findings suggested that occupational therapists working in acute settings can increase their confidence, enhance professional identity and further promote occupational therapy, through increasing the use of occupational language. Similarly, Gillen and Greber (2014), have suggested several strategies for occupational therapists in acute settings to make change in their practice. They recommended that therapists create more discussion about occupation and reframe the way they think and practice to be more focussed on an occupation-based approach. Furthermore, other authors have suggested that therapists incorporate occupational therapy models in acute settings to enhance an occupation-based approach (Maclean et al., 2012; Melton et al., 2008).

Overall, existing literature provides supporting evidence towards promoting change within acute settings to better reflect contemporary occupational therapy philosophies in practice. However, there is little research which explores acute occupational therapists' knowledge, skills and perceptions of contemporary occupational therapy philosophy and practice. For this research the term 'contemporary occupational philosophy and practice' is used to capture both historical and modern views of the profession and practice. This includes concepts such as Kielhofner's (2009) Contemporary Paradigm, occupational therapy models, Fisher's (2013) occupation-based, occupation-centred and occupation-focused taxonomy, and Gray's (1998) occupation as a means and an ends approach to practice. 
There is no existing research about understanding occupational therapists' perceptions of an acute service transformation towards contemporary occupational therapy philosophy and practice, which led to the development of this study. The acute occupational therapy department at a specialist paediatric hospital in metropolitan Australia, is in the very early stages of a service transformation to increase their alignment with contemporary occupational therapy philosophy and practice. This research was developed to provide the service managers with initial information regarding the participants perceptions of contemporary occupational therapy and identify what was needed for the next steps of the service transformation. Therefore, the aims of this study were:

a) To understand occupational therapists' knowledge and skills regarding contemporary occupational therapy philosophy and practice.

b) To understand occupational therapists' attitudes towards and motivation for a service transformation towards contemporary occupational therapy philosophy and practice.

\section{Method}

This study was located within the interpretive paradigm and followed a qualitative approach (Cresswell, 2014). Phenomenology was chosen as the research aimed to understand occupational therapists' lived experiences of a phenomenon (Creswell et al., 2007), namely, the early stages of the service transformation towards contemporary occupational therapy. The main purpose of phenomenology is to collect the views of a number of participants who have experienced the phenomenon and combine those experiences into a description of the universal essence of the experiences for all individuals (Creswell et al., 2007).

This study gained ethical approval from Griffith University Human Research Ethics Committee (Reference: 2017/028) and Children's Health Queensland Human Research Ethics Committee (Reference: HREC/16/QRCH/375). 


\section{Research participants}

The participants were occupational therapists working in the acute occupational therapy department at a specialist paediatric hospital in metropolitan Australia. Participants were recruited through a purposive sampling approach (Ritchie and Lewis, 2003) which began in December 2016 and continued until March 2017. Initial contact was made with potential participants via an in-service presentation which introduced the research. Occupational therapists were asked to volunteer by emailing the principal researcher (A.M). Eight occupational therapists volunteered to participate and all were recruited. All participants received an information sheet and signed a voluntary written consent form. All participants were female. Participants had varying years of occupational therapy experience (1-25 years) and were practising across a range of junior and senior positions. Additionally, participants worked in various areas of acute occupational therapy practice which meant a wide range of experiences and perspectives were explored. Pseudonyms have been used to ensure anonymity.

\section{Data Collection}

Data was collected over a two month period, using semi-structured interviews conducted by the principal researcher in a private room at the hospital. Each participant completed one 60-90-minute interview. A series of open-ended questions were asked during the interviews to encourage participants to share their individual experiences of practice as an occupational therapist in an acute setting and their perceptions of the service transformation towards contemporary occupational therapy. Other questions addressed therapists' knowledge, skills, attitudes and motivations regarding contemporary occupational therapy philosophy and practice. The interviews were semi-structured to allow participants to lead the 
conversation and share their experiences in detail, but also allowed the interviewer to guide questions as required (Clarke, 2009).

The interviews were audio-recorded and transcribed verbatim. The principal researcher transcribed two of the interviews and the remaining six were completed by a transcription company. All transcripts were compared to the original audio recordings to ensure accuracy. Finally, to comply with ethical requirements, the transcripts were deidentified by the principal researcher before being shared with other members of the research team.

\section{Data analysis}

The interview data was analysed using Braun and Clarke's (2006) six steps of thematic analysis. The first stage involved becoming familiar with the data and was achieved by repeatedly reading the transcripts, searching for meanings and patterns in the data. Key statements and quotes were highlighted and initial ideas noted. The next stage involved inductive coding, and so emerging codes were data-driven (Braun and Clarke, 2006). Coding was completed through a systematic approach which involved manually coding each individual transcript by analysing line by line and writing notes, as well as using highlighters to colour code specific patterns within the data (Braun and Clarke, 2006). During this stage, the principal researcher kept a reflective journal and actively collaborated with another member of the research team (A.D) on a regular basis to maximise trustworthiness, strengthen rigour and to mitigate any bias with coding. Once initial coding was complete, the principal researcher used Post-it Notes to collate all of the codes in order to search for possible themes; this is stage three. This involved transferring all codes onto coloured Post-it Notes and organising these on large flip charts into meaningful groups. Stage four of analysis was refining and reviewing themes through drawing thematic mind maps, and re-reading 
transcripts to ensure themes accurately represented the entire data set. Furthermore, two members of the research team (A.D, M.M) were involved in theme development to strengthen rigour. Stage five involved defining and naming the themes. The final stage of analysis was to generate an in-depth description of participants shared experiences and perspectives of the service transformation towards contemporary occupational therapy (Creswell et al., 2007).

\section{Findings}

Four main themes were identified from data analysis, these include: 1) a glimmer of occupation; 2) variability with recognising and articulating the core of occupational therapy; 3) therapists' externalising challenges; and 4) barriers and enablers to change.

\section{A glimmer of occupation}

Participants recognised the importance of occupation, and all appeared to address occupation in their practice in some way. However, their focus on occupation was inconsistent and in this way there was a glimmer of occupation in their work. Most therapists discussed occupations with their patients (however not always using the term 'occupation'), some thought about occupation, and others actively engaged patients in occupation during assessments and/or interventions.

During the initial stages of assessment, most participants described collecting information regarding their patients' occupational history. Several participants discussed utilising occupational therapy models, including the Person, Environment, Occupation model (Law et al., 1996) and the Canadian Model of Occupational Performance and Engagement (Polatajko et al., 2013), to guide their information gathering and assessment. Some therapists reported that occupation was their first thought or initial question with their patients, while others gathered procedural information about the injury or illness before seeking details about 
the patient's occupations. Three participants discussed using the occupation of play to assess their patients. Karen provided a good example of this and noted some of the benefits of occupation-based assessments:

...a less threatening and more relatable way by playing a game or doing some colouring or painting...rather than sitting face to face, doing an interview asking about their coping or their participation in different roles.

Further on from the assessment phase, some participants identified occupational issues with their patients and used these as the focus of goal setting and therapy, however the majority of participants focused therapy on non-occupational issues including adherence with treatment regimes and impairment specific protocols which were more biomedical in nature.

Moreover, some participants acknowledged that they did not always focus on occupations or practice a "top down approach". As a result, interventions were based on a biomedical perspective focussing on improving impairments, but with an end goal of returning to occupations. For example, Angela noted:

There's a big view 'yes let's have a look at the occupations and challenges that you're going through' but for a period of time we might actually need to look at some exercises and a particular protocol to be able to get back to doing and using your hand.

At times biomedical impairment focused interventions were prioritised over occupational therapy goals. Susan commented "sometimes we just need to address something really medicalised because that's where the child is at that moment and it's not until later that we can truly put on our [occupational therapy] hat in our [occupational therapy] role."

Although participants frequently talked about a range of occupations with their patients, they infrequently engaged patients in occupation. Consequently, most participants 
had a focus on occupation but did not consistently implement occupation-based practice. There were, however, some strong examples of therapists engaging patients in occupationbased interventions, most frequently play.

We really focused on his [the child's] play and the things that you would expect a little boy of his age - that would provide the therapy...but took the pressure off completely because she [the child's mother] just felt like she was playing with them. It wasn't just about doing exercises, it was about playing in an appropriate way and setting it up for [the mother] so she could do that. (Elizabeth)

Mary felt that occupation-based practice was more effective at improving patients' health and reported that patients often recovered faster when they are able to do their occupations. Additionally, several participants described occupation-based practice, in particular play, as being more motivating, engaging and meaningful for patients. Overall, it was clear that participants recognised the benefits of occupation-based practice and the value of occupation for health and wellbeing.

\section{Variability with recognising and articulating the core of occupational therapy}

Throughout the interviews, it was evident that participants were not consistently recognising and articulating the core foundations of the profession or using occupational language in their documentation or discussions with clients and colleagues. Some participants recognised that occupation was unique to occupational therapy and their contribution to the team was an occupational perspective, focusing on occupation and enabling occupational performance and engagement. However other therapists appeared more uncertain about the core domain of occupational therapy and had difficulty articulating their point of difference. For example, Angela identified family-centred practice as the core philosophy of occupational therapy. In addition, Dorothy noted: 
We understand child development and we understand what kids are motivated by, and how to get that best performance out of a child, and how to manipulate an environment, an activity, or a scenario - So I think that's what's unique about [occupational therapy].

Although the term 'best performance' may have been used instead of occupation, Dorothy did not specifically identify occupation as the profession's point of difference.

When asked to describe contemporary occupational therapy, participants provided a range of features including: family-centred practice, client-centred practice, occupation, a top down approach, being holistic, evidence-based practice, goal setting, occupational therapy models, outcome measures and building parent efficacy. Dorothy believed contemporary practice was about being "adaptable" and "fitting in with what else the rest of the team is providing the family." Interestingly, Karen was the only participant to mention occupationbased practice as a key feature of contemporary occupational therapy. Overall, family-centred practice was the most cited response from participants. Even though some participants identified occupation as core to the profession, there was a perception that contemporary occupational therapy was much more than this.

Participants' descriptions of occupational therapy also varied according to who they were speaking with. Differences were noted based on the person's previous experience with occupational therapy, their age, the specific referral request, and the ward/service. Several participants highlighted that the broad nature of occupational therapy and the different caseloads they worked in made it difficult to explain occupational therapy. There was a perception that occupational therapy was very different in different settings, for example acute versus community. This highlights that some participants did not recognise occupation as the core foundation and common thread across all areas of occupational therapy practice. 
During descriptions of occupational therapy, some therapists addressed the possible perception of others that what they did could look the same as what other professionals did with patients, but that they were likely to have a unique perspective. For example, Elizabeth said:

I would usually say that I'm part of this multidisciplinary team and that a lot of our things overlap there. So, the physio will be interested in how the baby is on their tummy, but I'll also be interested in that, but for potentially different reasons.

Although some participants used the term occupation when describing their practice, a wide range of other terms were used instead; activities, daily living skills, things, jobs, key performance areas, function, best performance, tasks, and roles. Several participants highlighted that the term occupation was not widely understood by others and was commonly seen only as employment. As a result, therapists often felt a "need to change the [language] for other people to be able to understand" (Angela). For example, some participants changed the language in their documentation to suit the medical team:

We need to write notes in a way that clearly gets the message across and medical professionals are really wanting to pick out keywords - sometimes we can lose that occupation, or we don't always fully detail the end outcome being improvement in occupational performance, or equally that medical professionals may not understand that language either. (Susan)

Furthermore, therapists had different attitudes towards using occupational therapy language. Several participants reported already making changes to their documentation to be more focused on occupation and said that it "has been really nice in being able to showcase to other disciplines what we do" (Dorothy). In contrast Angela stated: 
I am concerned that there is a risk if we use too much of our own [language] but nobody else understands - I feel really strongly that if we want to be a profession that's listened to then we need to be able to use [language] that's used by others, not just by ourselves.

Despite this, many participants believed that changing their language and documentation was the most important for the service transformation, and that this would lead to them practising in a contemporary manner. Elizabeth described her vision of the service transformation through the following quote;

If we wake up and then it had happened [the service transformation] and things have changed then I think we would see medical and other allied health professionals really understanding the word occupation and occupational performance, and that they would be using that language in the referrals that they make. I think we would see OTs more consistently using occupation in their assessment and their intervention and also in their documentation.

\section{Therapists' externalising challenges}

All participants were readily able to identify environmental and external barriers to them practicing in a contemporary manner, however few participants mentioned personal barriers. Several participants highlighted that the health service directed their practice through guidelines and policies. The fast paced, discharge focussed nature of the hospital was identified as another barrier to contemporary practice. All participants felt they had insufficient time to practice in a contemporary way. Some went as far as to suggest that focussing on occupation was "not possible" in an acute setting "when you're needing to keep up a pace." Overall, there seemed to be a perception that contemporary occupational therapy practice took more time, and this was not available. For example, Dorothy said: 
I've got 25 kids in the clinic, how do I go and assess the impact this injury is having on each of these children. And trying to really understand it from a family-centred occupational performance kind of framework, I don't have that time. Because that would take me all day.

In sharp contrast, Nancy felt that there was "quite a bit of time where we could even, I think, do a lot of contemporary practice." Nancy also added, "I don't think I would be too negatively impacted by it [time]... I don't think time would be something that will be a concern."

Participants believed that the largest single barrier within the practice context was their perception that the multidisciplinary team did not understand the occupational therapy contribution. As a result, participants felt that referrals to occupational therapy were not made because all of the "bottom-up components are being managed and so, [occupational therapy] may not be seen as somebody who can offer something." Within the acute environment, referrals were powerful in shaping occupational therapists' practice. Some participants believed that medically-focused referrals limited their ability to focus on occupation and influenced them to take a "bottom up approach." However, others felt even within this biomedical framework, they could broaden the scope of their practice to be more occupational than the initial referral might have suggested.

Participants were conscious of the multi-disciplinary team's views of their practice. They described being known as the "equipment lady" or the person who is "going to play with the patient or distract them during something." Elizabeth felt “...very conscious that sometimes it doesn't look like you're doing therapy or you don't look like you're doing much, whereas actually there's method behind it all." As a result, therapists felt as though they needed to justify their clinical reasoning for occupation-based practice to other health 
professionals. Overall, participants believed that increasing the multi-disciplinary teams understanding would enable them to practice in a contemporary manner as this was perceived as the main problem stopping them from practising in this way. Nancy added:

In an ideal world for [occupational therapy] to practice contemporary would be a lot more easily facilitated if doctors knew... and understood our perspective because we understand theirs is very medical but, I think there's a lot of work that needs to be done.

Interestingly, Nancy later discussed how educating the multi-disciplinary team was a key role for occupational therapists' commenting:

It's the role of [occupational therapist]'s to really bring that home... using really occupational based language in [our] notes. So, not just saying 'review for cooking session' but a 'review for engagement in meaningful occupation's and translation of occupations to home or engagement in various roles at home.'

\section{Barriers and enablers to change}

Participants generally had a positive attitude towards the service transformation, with most commenting that they felt excited, passionate and supportive. This suggests that most participants were willing and motivated to move towards aligning their practice with contemporary occupational therapy. Participants commented: "It's exactly what we should be doing" and "It's really core to us and should be brought back to the forefront." Furthermore, it was evident that the power of occupation and occupational therapy was a key motivator for some participants. Several therapists described how proud they were to be a part of the profession: "I just love [occupational therapy] and what we could offer, and I love the models and I think that it's a really unique point of difference.” Additionally, some participants described feeling more at ease when their practice was focused on occupation. Brenda said; 
“Once I started using those start points [occupation], that helped define me, helped me feel more comfortable about the role that I had to offer." Furthermore, Susan suggested that focussing on occupation "would help to boost [occupational therapists]' confidence" as it is the core domain and that it had the potential to;

...reduce that feeling of anxiety or stress about working in an unfamiliar caseload because at the end of the day, we're focusing on occupation and, yes there's particular skills that we need to develop but we're asking about occupation and that's what we're all really good at doing.

Participants also anticipated increased work satisfaction as an outcome of practising in a contemporary manner. Brenda stated, "I hope it helps me to enjoy my work more. I feel proud of what we do." Furthermore, Mary hoped that she would have a better connection to the profession in terms of "holding onto actually why I became an [occupational therapist] in the first place."

Although participants expressed their motivation and discussed potential benefits of the service transformation, many anticipated barriers to change within their current workplace. Therapists' resistance to change was raised by several participants, however all hoped that majority of the team were motivated for the service transformation. Some participants highlighted that change can bring about a level of anxiety. Furthermore, participants mentioned the potential for people to not engage with the service transformation, particularly those who believed there was no room for improvement. This was evident as some therapists thought their practice was already contemporary and were unsure what changes could be made. Some participants perceived that people would struggle to adapt to a new way of practice and being less biomedical in their focus. Brenda believed there were “people who've built up expertise in certain areas and technical skills that they feel reluctant 
to let go of and I think there's always going to be people who don't [engage]." Additionally, there was evidence of participants being unwilling to lose a biomedical perspective:

What won't change is the interventions that we're offering, because there's a lot evidence. The way to manage [this condition] is through very specific [biomedical treatment]. That is the clinically proven way in which you do that. That won't change the fact that those interventions still need to happen and they're appropriate within this setting, given they're highly specialised. (Dorothy)

Consequently, this therapist appeared to prioritise evidence-based practice and biomedical treatments over contemporary practice. Notably, some therapists suggested that it was "easier" to focus on the biomedical view versus focusing on occupation. Elizabeth noted:

You can get a referral that says well we want [occupational therapy] to do this thing, and you're like - 'well, that's not really what we do.' But also, sometimes it's easier I think when you're strapped for time and things are complicated to just go in and do the ranging and positioning and just leave.

Therefore, some therapists conformed to the medical focused referral as it was the "easier" option, even though they perceived it as an inappropriate referral.

Dorothy believed that it would be difficult to change practice as specific roles for occupational therapy were already established based on certain clinical tasks. Furthermore, some participants anticipated that there would be criticism from the medical team. Elizabeth believed that areas "where therapists traditionally work in a more bottom-up approach, the doctors will go, 'What do you mean you don't do this?' and that there could be some backlash in that respect." Overall, participants hoped that the service transformation would lead to further recognition of occupational therapy within the hospital and identified a need to broaden the multi-disciplinary team's understanding of their contribution. Although 
participants acknowledged how vital promotion was "in order to survive", some participants were not as motivated to advocate for their role. Dorothy commented that "it depends what day it is and how much energy I've got for it to be honest...there's various times in my career in which I've had more energy to try and explain it than not." In addition, Elizabeth admitted having difficulty knowing how to promote occupational therapy to the medical team when therapy “didn't look like much." Consequently, some therapists might choose not to advocate for occupational therapy and occupation-based practice because they are worried about how they will be perceived by other health professionals or they may not have the knowledge or skills to advocate for the profession.

Moreover, several participants highlighted a desire for further knowledge and skills regarding contemporary occupational therapy, particularly occupational therapy models and occupation-based practice. Some therapists commented on the fact that they did not learn about these concepts at university or had not looked at them for a long time, perhaps indicating that they did not prioritise continuing their learning in this area. It appeared that the preferred method of learning was through in-services and guest speakers as they believed this would generate more discussion about contemporary occupational therapy within the team. Furthermore, most participants suggested that education have a practical component involving case examples so that therapists could easily see how a contemporary approach applies in their practice.

\section{Discussion and implications}

In light of the above results, the next section will outline three key elements which relate to practical implications for occupational therapists'. These include: 1) taking responsibility, 2) changing language and practice, and 3) harnessing occupation as the core 
domain. Finally, recommendations will be made for future research in this area and study limitations will be highlighted.

\section{Taking responsibility}

Participants frequently discussed the challenges they experienced practising in a contemporary manner, with most of the challenges being external to the therapist. This is consistent with existing literature which often highlights external barriers including; the fastpaced discharge focused culture (Britton et al., 2016; Keesing and Rosenwax, 2011), workplace expectations (Aiken et al., 2011; Estes and Pierce, 2012), the dominant biomedical model (Ashby et al., 2015; Wilding and Whiteford, 2007), and feeling undervalued and misunderstood by the multi-disciplinary team (Ashby et al., 2015; Kinn and Aas, 2009; Wilding and Whiteford, 2007). Furthermore, all participants emphasized limited time and increased pressure as a barrier to contemporary practice. Although, it is worth reflecting on how much choice therapists have in how they structure their time with patients. Some authors contend that thinking, talking and acting with a focus on occupation is ultimately an individual decision of the therapist and should not be restrained by contextual factors (Gillen and Greber, 2014; Gustafsson et al., 2014; Joosten, 2015). Hence, despite challenges, it is vital for therapists in acute settings to explicitly articulate the value they place on contemporary occupational therapy philosophies in a way which is visible within their practice.

if therapist in acute settings really value contemporary occupational therapy philosophies, they should work towards better reflecting this in their practice.

Furthermore, there was a general perception among participants that increasing the multi-disciplinary team's understanding of their role would lead to occupational therapists practising in a contemporary manner. Therapists did not always acknowledge their own role 
in promoting occupational therapy or being consistent with what they say they do and what others see them do. This could be seen as therapists externalising the problem and abdicating responsibility to others, and not taking responsibility for their own knowledge and understanding.

\section{Changing language and practice}

Participants' language was inconsistent when describing occupational therapy and articulating the core focus of the profession, which is congruent with existing literature (Aiken et al., 2011; Kinn and Aas, 2009; Wilding and Whiteford, 2007; Wilding and Whiteford, 2008). Interestingly, participants introduced the terms 'top-down' and 'bottomup' without prompting from the interviewer. Trombly (1993: 253), defined a bottom-up approach as "assessment and treatment that focuses on the deficits of components of function, such as strength, range of motion, balance,... which are believed to be prerequisites to successful occupational performance or functioning." In contrast, described a top-down assessment as "one that starts with inquiry into role competency and meaningfulness,.. . [and] further determines which particular tasks define each of the roles . . whether he or she can now do those tasks, and probable reasons for an inability to do so".

The frequent use of 'top down' and 'bottom up' suggests these terms were commonly used by the therapists to describe their practice.

Overall, the variation in descriptions could suggest a potential gap in knowledge regarding contemporary occupational therapy. As without an accurate understanding of contemporary occupational therapy, therapists are likely to have difficulty articulating it to others, which may be the reason why the profession has continued to struggle with maintaining a strong professional identity (Hooper and Wood, 2002; Turner and Knight, 2015). This was hinted at in the results as some participants expressed wanting to know more 
about occupational therapy models. However, this was not emphasised as a major contributing factor for participants. This perhaps further reinforces the limited acknowledgment of personal control participants felt they had to implement change.

Furthermore, there was a perception among participants that changing language and documentation to be more focused on occupation would lead to contemporary occupational therapy practice. Similarly, Wilding and Whiteford (2009) reported that occupational therapist's assessments and interventions became more focused on occupation when they began using more occupational language, suggesting that changing language may lead to a change in behaviour. However, it is likely that more than a change in language is needed, and therapists also need to focus on consistently incorporating occupation into their practice, as there is limited evidence of occupation-based practice within acute settings (Di Tommaso et al., 2016; Keesing and Rosenwax, 2011; Mulligan et al., 2014; Rogers, 2007).

Despite valuing and recognising the many benefits of an occupation-based approach, therapists in this research often focused on biomedical interventions with an end goal of returning to occupational performance and engagement, which is consistent with $\mathrm{Di}$ Tommaso et al.'s (2016) research. It is suggested that this problem may be attributed to a lack of knowledge and skills regarding occupation-based practice, which has been supported by several authors (Di Tommaso et al., 2016; Keesing and Rosenwax, 2011).

\section{Harnessing occupation as the core domain}

Some participants in this research highlighted occupation as a key component of contemporary occupational therapy, however the most cited response was family-centred practice. This may suggest that participants perceived that it was more important to focus on family-centred practice than occupation-based practice. The emphasis on family-centred 
practice may be attributed to the paediatric nature of the hospital where the research was conducted, where this concept is a recognised value and priority of the organisation. These findings however are consistent with Di Tommaso et al.'s (2016) research where some occupational therapists in acute settings indicated that client-centred practice was prioritised over an occupation-based approach. Nevertheless, client-centred and family-centred practice are not necessarily specific to occupational therapy, as many other health professionals consider having this approach. Several therapists in the research study described themselves as being "similar to" other health professionals, which clearly contributes to misunderstanding of occupational therapy. As a result, the profession needs to define its role and justify the point of different in order to survive in the competitive healthcare system. Furthermore, occupational therapists need to take responsibility with consistently articulating occupation as their unique contribution and ensuring their practice is reflective of contemporary occupational therapy philosophy. If therapists continue to be unsure of their core focus and are unable to implement occupation-based practice, clients could end up being deprived of our unique expertise regarding the importance of occupation for health and wellbeing (Fortune, 2000; Gustafsson et al., 2014).

\section{Implications for practice}

Without a firm understanding of the core foundations and philosophies of the profession, it will be difficult for therapists to articulate this to others or make changes to their practice. This research found that therapists' lack of knowledge regarding the core foundations of the profession, impacts on whether their practice is aligned with contemporary occupational therapy. Occupational therapists should therefore prioritise knowledge acquisition, as this study highlighted that therapists did not foresee their own knowledge as a significant impacting factor on the service transformation. This is a challenge for the profession, if therapists do not recognise their own knowledge limitations, they are unlikely 
to take the needed steps to overcome this issue. This may further contribute to therapists feelings a lack of control and externalising the barriers to service transformation. Therefore, more emphasis should be placed on furthering occupational therapists' knowledge of contemporary occupational therapy philosophies and the skills to apply this in practice.

Therapists must take responsibility for broadening their own knowledge and skills regarding contemporary occupational therapy. Gillen and Greber (2014) suggested therapists extend their knowledge through provision of practice education for students and through participating in professional development opportunities relating to contemporary occupational therapy philosophy, rather than technical skills in specialised areas of practice (Ashby et al., 2013; Fortune, 2000; Kinn and Aas, 2009). Therefore, therapists must be proactive and motivated to learn about the unique philosophies of the profession.

A broader strategy suggested by several authors is becoming a community of practice, which has been defined as "groups of people who share a concern or a passion for something they do and learn how to do it better as they interact regularly" (Wenger and Wenger, 2015: 1). Therefore, occupational therapists could join as a community of practice with a shared focus on occupation and ensure they are reflecting contemporary occupational therapy philosophies in practice (Turner and Knight, 2015). A good example of this is Wilding and Whiteford's (2009) research study, which provided supporting evidence of occupational therapists becoming a community of practice and changing their language within an acute setting.

\section{Recommendations for future research}

Evidently, this research has highlighted a gap in relation to therapists' knowledge of contemporary occupational therapy philosophy. For the next stage of the acute department service transformation, it is recommended that an education program be implemented in 
order to increase therapists' knowledge and understanding of contemporary occupational therapy philosophy. Therefore, future research should explore the process of change and knowledge translation practices, in order to evaluate whether increasing therapists' knowledge regarding contemporary occupational therapy is effective at facilitating change within the profession.

Additionally, an alternate means of exploring the change process could be Participatory Action Research (PAR). PAR is a methodology based on reflection and action, which aims to address specific problems and directly involves those who are taking actions to improve the problem (Baum et al., 2006). Within this methodology, participants determine what they need to support change with the facilitation of the external researchers, which may be a beneficial model in terms of ensuring a sustainable service transformation, and help participants to reflect on their own knowledge development areas.

Although an aim of this study, future research should also further explore occupational therapists' attitudes and motivation for considering a service transformation within a variety of settings and with a larger population, as this will be key in driving universal change for the profession. Overall, this research paper has highlighted several concerns for the profession which could be further explored through PAR.

\section{Study limitations}

Due to the qualitative nature of this study, the findings cannot be widely generalised to all occupational therapy settings, however they may be valid for similar acute settings within the Australian context. Additionally, this study only captured participants perspectives at one time point, therefore, participants' experience of change over time cannot be drawn from these results. Another limitation was that participants were volunteers and so these findings may be only reflective of those with an interest/commitment to contemporary 
occupational therapy. Hence, therapists who may not have an interest in or specific knowledge of contemporary occupational therapy, may have provided different responses regarding their perspectives of the service transformation. However, all participants had varying levels of experience and differing viewpoints regarding contemporary occupational therapy which was captured during their individual interviews.

\section{Conclusion}

The findings from this study contribute to the existing body of literature regarding contemporary occupational therapy philosophy and practice in acute settings, with a specific focus on whole service transformation in a paediatric hospital. These findings highlight that therapists in acute settings are attempting to incorporate occupation in their practice and many therapists have positive attitudes towards contemporary occupational therapy. Furthermore, the majority of occupational therapists recognise the power of occupation and the benefits of an occupation-based approach, however the implementation of occupationbased practice appears to be inconsistent in acute settings due to a range of perceived external barriers. Occupational therapists must take responsibility for incorporating more occupationbased practice and advocating for the profession with a unified focus on occupation, using occupational language. However, there also seems to be a need to enhance occupational therapists' knowledge of contemporary occupational therapy philosophy and improve their skills to implement this in practice. 


\section{Key Findings}

- Occupational therapists in acute settings are attempting to incorporate contemporary philosophies, however the implementation of occupation-based practice is inconsistent.

- Need for further knowledge and skills regarding contemporary philosophies and practice.

\section{What the study has added}

Occupational therapists are open to transforming practice however, perceived external challenges and lack of knowledge may hinder the effective implementation of occupationfocused practice change in acute settings. 


\section{Statements:}

\section{Research Ethics}

Ethical approval was obtained from Griffith University Human Research Ethics Committee (Reference: 2017/028) and Children's Health Queensland Human Research Ethics Committees in December 2016 (Reference: HREC/16/QRCH/375; SSA Reference Number: $\mathrm{SSA} / 17 / \mathrm{QRCH} / 40)$.

Consent

All participants provided written informed consent to be interviewed and recorded for this study.

\section{Declaration of conflicting Interests}

The primary researcher conducted this study as a student occupational therapist, therefore had no conflicts of interest during data collection, analysis or when drafting the results. The primary researcher later became an employee at the place of research after the study was completed.

\section{Funding}

The authors received no financial support for the research, authorship or publication of this article.

\section{Contributorship}

Alice Murray completed initial project scoping, literature searching, development of research design, data collection, data analysis and manuscript preparation, under supervision of the following authors. Dr Amelia Di Tommaso and Professor Matthew Molineux supervised 
Alice Murray and contributed to initial project scoping, literature searching, development of research design, data collection, data analysis and manuscript preparation. Anna Young and Penny Power were clinician partners on this project and provided supervision and input into initial project scoping, literature searching, development of research design and manuscript preparation. Final approval of the version to be published was sought from all authors, with agreement to be accountable for all aspects of the work.

Acknowledgements

Not applicable. 


\section{References}

Aiken FE, Fourt AM, Cheng IK, et al. (2011) The meaning gap in occupational therapy: Finding meaning in our own occupation. Canadian Journal of Occupational Therapy 78: 294-302.

Ashby S, Gray M, Ryan S, et al. (2015) Maintaining occupation-based practice in Australian mental health practice: A critical stance. British Journal of Occupational Therapy 78: 431-439.

Ashby S, Ryan S, Gray M, et al. (2013) Factors that influence the professional resilience of occupational therapists in mental health practice. Australian Occupational Therapy Journal 60: 110-119.

Baum F, MacDougall C and Smith D. (2006) Participatory action research. Journal of Epidemiology and Community Health 60: 854-857.

Braun V and Clarke V. (2006) Using thematic analysis in psychology. Qualitative Research in Psychology 3: 77-101.

Britton L, Rosenwax L and McNamara B. (2016) Occupational therapy in Australian acute hospitals: A modified practice. Australian Occupational Therapy Journal 63: 257-265.

Clarke C. (2009) An introduction to interpretative phenomenological analysis: A useful approach for occupational therapy research. British Journal of Occupational Therapy 72: 37-39.

Cresswell J. (2014) Research design: Qualitative, quantitative, and mixed methods approaches Thousand Oaks, CA: SAGE Publication Ltd.

Creswell JW, Hanson WE, Clark Plano VL, et al. (2007) Qualitative research designs: Selection and implementation. The Counseling Psychologist 35: 236-264.

Di Tommaso A, Isbel S, Scarvell J, et al. (2016) Occupational therapists' perceptions of occupation in practice: An exploratory study. Australian Occupational Therapy Journal 63: 206-213.

Estes J and Pierce DE. (2012) Pediatric therapists' perspectives on occupation-based practice. Scandinavian Journal of Occupational Therapy 19: 17-25.

Fisher A. (2013) Occupation-centred, occupation-based, occupation-focused: Same, same or different? Scandinavian Journal of Occupational Therapy: 162-173.

Fortune T. (2000) Occupational Therapists: Is our therapy truly occupational or are we merely filling gaps? British Journal of Occupational Therapy 63: 225-230.

Gillen A and Greber C. (2014) Occupation-focused practice: challenges and choices. British Journal of Occupational Therapy 77: 39-41.

Gustafsson L, Molineux M and Bennett S. (2014) Contemporary occupational therapy practice: the challenges of being evidence based and philosophically congruent. Australian Occupational Therapy Journal 61: 121-123.

Hooper B and Wood W. (2002) Pragmatism and structuralism in occupational therapy: The long conversation. American Journal of Occupational Therapy 56: 40-50.

Joosten AV. (2015) Contemporary occupational therapy: Our occupational therapy models are essential to occupation centred practice. Australian Occupational Therapy Journal 62: 219222.

Keesing S and Rosenwax L. (2011) Is occupation missing from occupational therapy in palliative care? Australian Occupational Therapy Journal 58: 329-336.

Kielhofner G. (2009) Conceptual foundations of occupational therapy practice Philadelphia, PA: F. A. Davis.

Kinn LG and Aas RW. (2009) Occupational therapists' perception of their practice: A phenomenological study. Australian Occupational Therapy Journal 56: 112-121.

Law M, Cooper B, Strong S, et al. (1996) The person-environment-occupation model: A transactive approach to occupational performance. Canadian Journal of Occupational Therapy 63: 9-23.

Maclean F, Carin-Levy G, Hunter H, et al. (2012) The usefulness of the Person-EnvironmentOccupation Model in an acute physical health care setting. British Journal of Occupational Therapy 75: 555-562. 
McCluskey A and Cusick A. (2002) Strategies for introducing evidence-based practice and changing clinician behaviour: A manager's toolbox. Australian Occupational Therapy Journal 49: 63-70.

McLaughlin-Gray J. (1998) Putting occupation into practice: Occupation as ends, occupation as means. The American Journal of Occltpational Therapy 52: 354-364.

Melton J, Forsyth K, Metherall A, et al. (2008) Program redesign based on the Model of Human Occupation: Inpatient services for people experiencing acute mental illness in the UK. Occupational Therapy In Health Care 22: 37-50.

Mulligan S, White BP and Arthanat S. (2014) An examination of occupation-based, client-centered, evidence-based occupational therapy practices in New Hampshire. OTJR Occupation, Participation and Health 34: 106-116.

Parnell T and Wilding C. (2010) Where can an occupation-focussed philosophy take occupational therapy? Australian Occupational Therapy Journal 57: 345-348.

Polatajko HJ, Davis J, Stewart D, et al. (2013) Specifying the domain of concern: Occupation as core. In: Townsend EA and Polatajko HJ (eds) Enabling occupation II: Advancing an occupational therapy vision for health, well-being, \& justice through occupation. Ottawa: CAOT Publications ACE, 13-36.

Ritchie J and Lewis J. (2003) Qualitative research practice: A guide for social science students and researchers, London: SAGE Publications.

Rogers S. (2007) Occupation-based intervention in medical settings. OT Practice, 12.

Trombly C. (1993) Anticipating the future: Assessment of occupational function. American Journal of Occupational Therapy 47: 253-257.

Turner A and Knight J. (2015) A debate on the professional identity of occupational therapists. British Journal of Occupational Therapy 78: 664-673.

Wenger E and Wenger B. (2015) Introduction to communities of practice: A brief overview of the concept and its uses. Available at: http://wenger-trayner.com/introduction-to-communitiesof-practice/.

Wilding C and Whiteford G. (2007) Occupation and occupational therapy: Knowledge paradigms and everyday practice. Australian Occupational Therapy Journal 54: 185-193.

Wilding C and Whiteford G. (2008) Language, identity and representation: occupation and occupational therapy in acute settings. Australian Occupational Therapy Journal 55: 180-187.

Wilding C and Whiteford G. (2009) From practice to praxis: reconnecting moral vision with philosophical underpinnings. British Journal of Occupational Therapy 72: 434-441.

Yerxa EJ. (1992) Some implications of occupational therapy's history for its epistemology, values, and relation to medicine. American Journal of Occupational Therapy 46: 79-83. 